\title{
Early detection of abnormalities in partial epilepsy using magnetic resonance
}

\author{
J H Cross, G D Jackson, B G R Neville, A Connelly, F J Kirkham, S G Boyd, M C Pitt, \\ D G Gadian
}

\begin{abstract}
The incidence of brain abnormalities determined by magnetic resonance in 30 consecutive children presenting with intractable complex partial seizures is reported. Images were optimised to visualise the hippocampus and cortical grey matter. Abnormalities of the hippocampus or temporal lobe were seen in all 19 children with clinical features of temporal lobe epilepsy and in six of the seven children with clinically unlocalised epilepsy. By contrast, in the four children with a clinical diagnosis of extratemporal epilepsy, no temporal or hippocampal abnormalities were seen. Generalised cortical abnormalities of uncertain significance were found in a total of 14 children from all groups. The identification of focal brain abnormalities using optimised magnetic resonance imaging enables early non-invasive assessment of children with intractable seizure disorders and the identification of patients for whom epilepsy surgery may be appropriate. It may also lead to a better understanding of the structural basis of intractable epilepsy, and thereby contribute to early treatment decisions.
\end{abstract}

(Arch Dis Child 1993; 69: 104-109)

Epilepsy in childhood is associated with signifcant cognitive and psychosocial morbidity. Although the majority respond favourably to anticonvulsant medication, up to $25 \%$ are intractable. ${ }^{12}$ If strict clinical and neurophysiological criteria are used to exclude benign syndromes, partial epilepsies may be intractable in $80-90 \%$ of patients. ${ }^{34}$ Early surgery may improve the outcome in these patients, particularly those with temporal lobe epilepsy.

In adults with intractable temporal lobe epilepsy, surgical temporal lobe specimens show hippocampal sclerosis in $50-65 \%$ of cases, foreign tissue lesions in $24 \%$, and cortical dysplasia in $4-6 \%$ of cases. ${ }^{6-8}$ The finding of such pathology in surgical specimens correlates with good postsurgical seizure control, ${ }^{9-11}$ and magnetic resonance imaging (MRI) can be expected to detect these lesions preoperatively when optimised to show abnormalities of the hippocampus and cortical grey matter. ${ }^{12-14}$

Epilepsy surgery is performed less commonly in children, due both to a reluctance to use invasive seizure localisation procedures, and to the uncertainty of prognosis when no clear lesion is present. The prevalence of hippocampal and other structural abnormalities is therefore not as well established in childhood epilepsy. MRI now provides a means for the non-invasive detection of brain abnormalities that may underly the seizure disorder. A relationship between the presence of hippocampal sclerosis in adult and prolonged early childhood febrile convulsions has also been suggested, ${ }^{15}$ and the use of MRI in children offers a non-invasive means of exploring this in more detail.

This study examines the incidence of cerebral abnormalities using optimised MRI in children with complex partial seizures, and relates these to clinical and electroencephalographic localisation and the antecedent history of seizures. We regard this as an important step toward understanding the pathological basis of intractable epilepsy and its coexisting cognitive and behavioural deficits.

\section{Subjects and methods}

Thirty consecutive children, age range 0.9-15.5 years, presenting during a 12 month period to a tertiary referral centre for advice on management of complex partial seizures, underwent MRI examination as part of their investigation. The medical records were reviewed; the developmental history and a detailed account of seizures were extracted. Based on this retrospective summary, a clinical diagnosis of seizure localisation and lateralisation was made independently by two paediatric neurologists blind to the findings on MRI. Consensus diagnosis was reached in all cases. All children in the present study were considered to have evidence of complex partial seizures at the time of assessment based on evidence of a focal onset progressing to impairment of consciousness, a distinct independent aura, a prolonged prodromal phase leading into the seizure, or focal features in the postictal period.

Both clinicians were asked to classify seizures as temporal, extratemporal, or of undetermined localisation. Temporal localisation was accepted if there was any one of the following: oroalimentary automatisms, stereotyped complex automatisms with postictal confusion, psychic aura such as fear or déjà $v u$, an aura of a formed auditory hallucination, a distinct epigastric aura, speech impairment before or immediately after the seizure, or non-specific aura or automatisms followed by confusion postictally. If there were features at the onset such as focal motor or sensory phenomena or rapid onset and cessation of the seizure, an extratemporal localisation was diagnosed. Seizures were considered as unlocalised if the prodrome could not be clearly defined or if consciousness was impaired at the start and the seizure had no specific localising features. Lateralisation of the seizure was considered 
Table 1 MRI findings related to clinical diagnosis

\begin{tabular}{|c|c|}
\hline Diagnosis & $\begin{array}{l}\text { No(\%) } \\
\text { subjects }\end{array}$ \\
\hline \multicolumn{2}{|l|}{ Temporal lobe epilepsy $(n=19)$} \\
\hline Temporal foreign tissue lesion & $3(16)$ \\
\hline Hippocampal abnormality & $11(58)$ \\
\hline \multicolumn{2}{|l|}{ Hippocampal abnormality alone (3) } \\
\hline \multicolumn{2}{|l|}{ With temporal neocortical abnormality (3) } \\
\hline \multicolumn{2}{|c|}{$\begin{array}{l}\text { With widespread abnormality }+/- \text { temporal } \\
\text { neocortical abnormality }(5)\end{array}$} \\
\hline Temporal neocortex abnormality & $5(26)$ \\
\hline \multicolumn{2}{|l|}{ Unlocalised epilepsy $(n=7)$} \\
\hline Hippocampal abnormality & $5(71)$ \\
\hline \multicolumn{2}{|l|}{ Hippocampal abnormality alone (1) } \\
\hline \multicolumn{2}{|c|}{ With temporal neocortical abnormality (2) } \\
\hline \multicolumn{2}{|c|}{$\begin{array}{l}\text { With widespread abnormality }+/- \text { temporal } \\
\text { neocortical abnormality }(2)\end{array}$} \\
\hline Temporal neocortex abnormality & $1(14)$ \\
\hline Generalised cortical abnormality alone & $1(14)$ \\
\hline \multicolumn{2}{|l|}{ Extratemporal epilepsy $(n=4)$} \\
\hline Focal cortical abnormality & $1(25)$ \\
\hline Cerebellar atrophy & $1(25)$ \\
\hline Widespread cortical changes & $2(50)$ \\
\hline
\end{tabular}

separately, and was based on any suggestion of lateralised motor phenomena or speech disturbance before or immediately after the seizure.

All electroencephalography (EEG) was recorded with Grass 8 or 12 channel EEG machines using previously described techniques. ${ }^{16}$ In 29 patients, conventional surface EEG had been performed within six months of MRI, and were reviewed independently by two clinical neurophysiologists. Lateralisation and localisation of slow waves or epileptiform spikes or sharp waves was noted. Discharges were considered to be multifocal when seen independently in more than one region and generalised when seen in a widespread distribution. Similar details were noted in ictal recordings.

All children underwent magnetic resonance imaging using a 1.5 Tesla Siemens system with a $25 \mathrm{~cm}$ circularly polarised head coil used as both transmitter and receiver. Children were sedated before scanning in accordance with a standard protocol. ${ }^{17}$ In order to visualise the mesial temporal structures optimally, inversion recovery images were obtained in planes parallel with and orthogonal to the long axis of the hippocampus. In both the tilted axial and tilted coronal planes, 10 slices of $5 \mathrm{~mm}$ were obtained with TR $2500 \mathrm{~ms}$, TI $300 \mathrm{~ms}$, TE $26 \mathrm{~ms}$, and a slice gap of $2.5 \mathrm{~mm}$. An asymmetric field of view was used with a matrix of $256 \times 135$ to reduce the imaging time to 7.9 minutes per sequence. In addition, images covering the whole brain were acquired using a double echo short tau inversion recovery sequence (TR $4000 \mathrm{~ms}$, TI $145 \mathrm{~ms}$, TE 23 , and $85 \mathrm{~ms}$, slice thickness $5 \mathrm{~mm}$, slice gap $2.5 \mathrm{~mm}$ ).

Images were reviewed blind to all clinical and electrophysiological data. The criteria used for the diagnosis of hippocampal abnormality were unilateral hippocampal atrophy, loss of internal structure, decreased signal on inversion recovery images, and increased signal on $\mathrm{T} 2$ weighted images. ${ }^{1218}$ If there was evidence of both a morphological abnormality and signal abnormality of the hippocampus, hippocampal sclerosis was diagnosed. Generalised cortical abnormalities included double cortex, thickened or irregular cortex, abnormalities of gyral pattern, significant atrophy, or signal abnormalities. ${ }^{1320}$ Abnormality of the temporal neocortex was diagnosed if there was significant atrophy, abnormal signal characteristics or significantly thickened grey matter with loss of definition of the grey-white matter junction. Foreign tissue lesion included any mass lesion such as a hamartoma, glioma, or dysembryoplastic neuroepithelial tumour.

\section{Results}

Of the 30 children, 16 had complex partial seizures alone (five of whom had episodes of

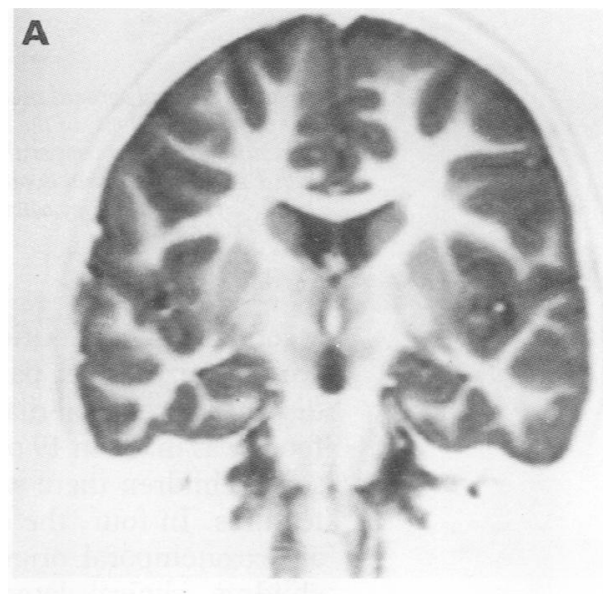

B
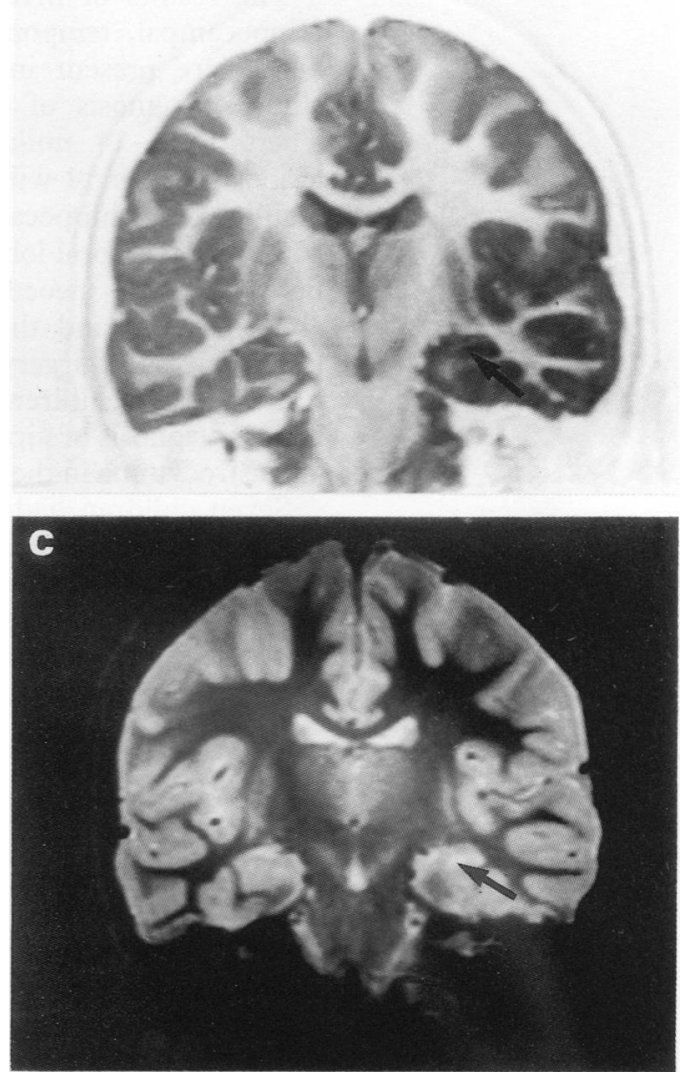

Figure 1 (A) Tilted coronal inversion recovery (TE $26 \mathrm{~ms}$, TI $300 \mathrm{~ms}$, TR $3500 \mathrm{~ms}$ ) showing the symmetry of the hippocampi in a normal child. (B) Tilted coronal inversion recovery (TE $26 \mathrm{~ms}$, TI $300 \mathrm{~ms}$, TR $3500 \mathrm{~ms}$ ) showing left hippocampal sclerosis (arrow) in a 12 year old girl who had a febrile seizure with right Todd's paresis at 14 months of age. Her habitual temporal lobe seizures began at age seven. The left hippocampus (arrow) is smaller than the right, with loss of defined internal structure, and TI weighted signal

hypointensity. (C) Tilted coronal T2 weighted DE-STIR image (TE $85 \mathrm{~ms}, T I 145 \mathrm{~ms}, T R 4000 \mathrm{~ms}$ ) showing increased $T 2$ weighted signal intensity in the left hippocampus (arrow). 


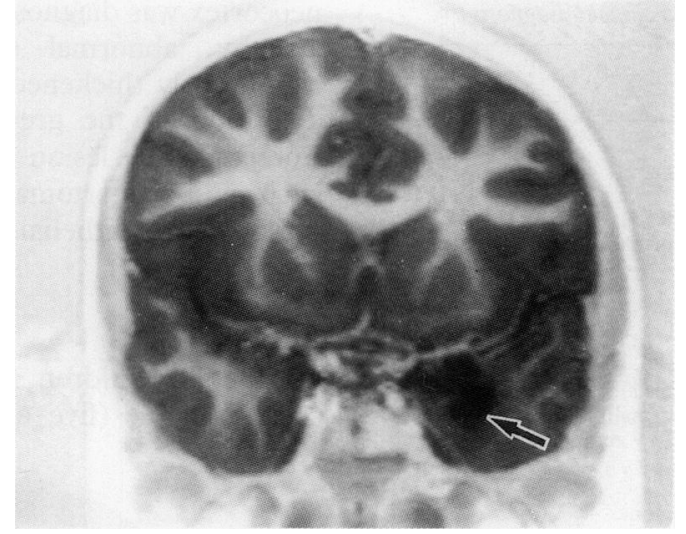

Figure 2 Tilted coronal inversion recovery $(T E 26 \mathrm{~ms}, T I$ $300 \mathrm{~ms}, T R 3500 \mathrm{~ms}$ ) of the temporal lobe of an 11 year old girl with intractable temporal lobe seizures. There is a lesion with $T 1$ weighted signal hypointensity in the left temporal lobe (arrow). Histopathology after temporal lobectomy showed a grade II astrocytoma.

secondary generalisation) and 14 had complex partial seizures as part of a multiple seizure disorder. A clinical diagnosis of a temporal lobe focus was made in 19 of the total group of 30 . In seven children there were no definite localising features. In four, the clinical history suggested an extratemporal origin. In 11 of the total 30 children clinical lateralisation of the seizure focus was suggested.

The results of MRI are shown in table 1 . Hippocampal, temporal lobe abnormalities, or both were present in all 19 children with a clinical diagnosis of temporal lobe epilepsy. There were 14 unilateral and five bilateral lesions. Of the 14 with unilateral MRI abnormality, six had hippocampal sclerosis (fig 1), four had other temporal lobe abnormalities, one had both temporal neocortical and hippocampal abnormalities, and three had a foreign tissue lesion (fig 2). Surgery was subsequently performed in two children and confirmed the radiological diagnosis of hippocampal sclerosis in one and astrocytoma in the other.

Seven children had complex partial seizures without localising features. Of these, five had a hippocampal abnormality, one had atrophy of the right temporal lobe, and one had widespread cortical change with no apparent
A

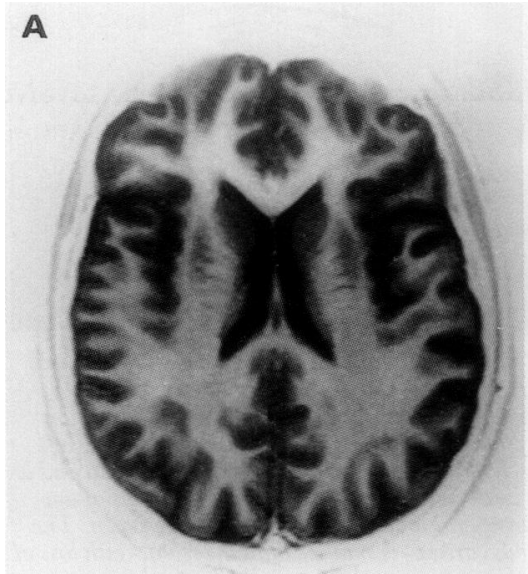

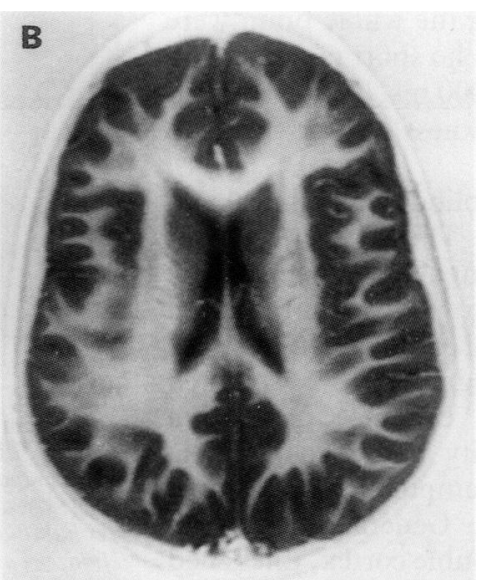

Figure 3 Tilted axial inversion recovery (TE $26 \mathrm{~ms}, T I 300 \mathrm{~ms}$, TR $3500 \mathrm{~ms}$ ) of a normal child $(A)$ and of a child with intractable frontal lobe seizures $(B)$. There is a generalised abnormality of the cortical gyral pattern in the child with epilepsy. focal pathology. Of the five patients with hippocampal abnormality, two had unilateral hippocampal sclerosis, two showed bilateral hippocampal abnormality, and the other showed progression from unilateral to bilateral hippocampal abnormality over six months.

No focal temporal lobe abnormality was seen in any of the four children with a clinical diagnosis of extratemporal epilepsy. Two children with clinical features suggestive of frontal lobe epilepsy had widespread cortical change with abnormal cortical thickness, sulcal pattern or both (fig 3). One child with focal motor seizures had MRI findings suggesting a neuronal migration defect of the corresponding parietal cortex (fig 4). One child had no abnormality of the cerebral hemispheres but showed cerebellar atrophy; she had been receiving phenytoin since early infancy.

Of all patients with a clinical diagnosis of temporal lobe or unlocalised seizures (26 children), temporal lobe abnormalities were seen in 25 (96\%) which included the hippocampus in 16 $(62 \%)$. Sixteen per cent of children with temporal lobe epilepsy had foreign tissue lesions in the temporal lobe. Only one patient in the clinically non-localised group did not have focal temporal lobe pathology on MRI.

Fourteen children of the total study group showed widespread cortical abnormalities; in 11 in addition to focal MRI defined pathology and in three as the only MRI determined abnormality. Widespread cortical abnormality was most commonly seen in children with complex partial seizures as part of a multiple seizure disorder (64\%). In children with complex partial seizures alone this was seen in only $31 \%$.

The interictal EEG recordings in eight of the 29 children showed localised epileptiform features and there were lateralised changes in a further three. Ten of the interictal studies $(34 \%$ of the 29 patients) were normal, and eight (28\%) showed multifocal or generalised features. In addition ictal recordings were available in five children. Two showed localised features, one of which had also been localised on the interictal recording, and the remaining three were multifocal or generalised. A total of 12 children (41\%) therefore showed lateralised features, including nine $(31 \%)$ that were also localised.

Of the 12 children with lateralised features seen on EEG, seven had an imaging abnormality on the same side as the EEG lateralisation. One had a neocortical abnormality in the opposite temporal lobe. In this child the clinical seizure pattern suggested temporal localisation without lateralising features, the interictal EEG was normal, and the ictal EEG performed after drug withdrawal showed only sharp waves in the temporal lobe contralateral to the imaging abnormality. Six of the nine children whose EEG studies were normal, and three of the eight with multifocal abnormalities had a localised structural lesion on MRI. Therefore, only $31 \%$ had a lateralising and localising abnormality on EEG compared with $62 \%$ (that is 18 of the 29 who underwent EEG) who had a focal structural abnormality on MRI.

Because complex partial seizures are commonly seen in children as part of a multiple 


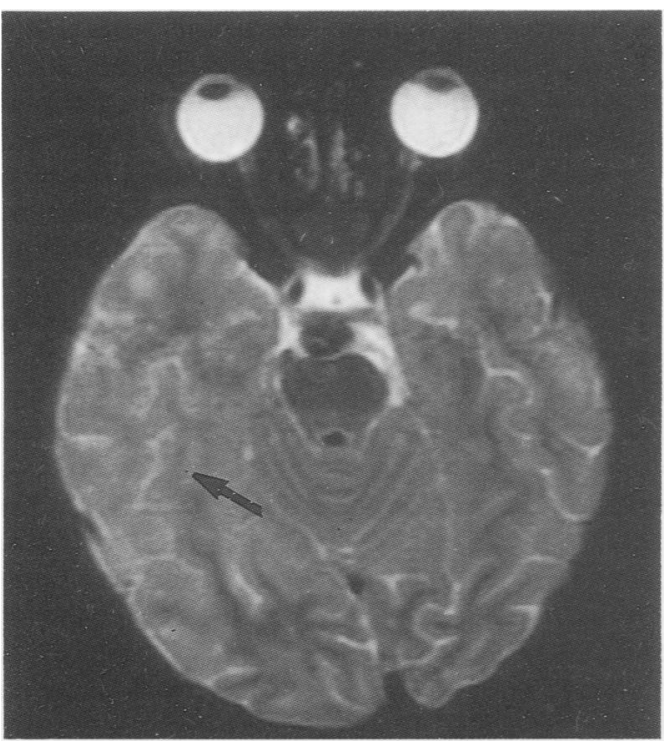

Figure 4 Tilted axial inversion recovery image (TE $26 \mathrm{~ms}$, TI $300 \mathrm{~ms}$, TR $3500 \mathrm{~ms}$ ) of an 18 month old child who presented with left focal motor status epilepticus. There is thickness of the grey matter and poor grey-white matter differentiation in the parietal region of the cortex in the right hemisphere suggesting an area of cortical dysplasia (arrowed).

seizure disorder, the 14 children in this series who fell into this category were considered separately. The EEG was normal in six of these, showed focal abnormalities in three, and multifocal abnormalities in five. The MRI showed isolated focal lesions in four of these children (one had a consistent focal abnormality on EEG and three had normal EEG). The remaining 10 children had either widespread MRI abnormalities, bilateral abnormalities or both, although four also had unilateral focal abnormalities. Thus eight of this group of 14 children $(57 \%)$ had a focal MRI abnormality compared with three (21\%) who had focal EEG. For comparison, in the children with complex partial seizures alone, $67 \%$ (10 of 15 ) had a focal MRI abnormality and $33 \%$ ( 5 of 15 ) a focal abnormality on EEG.

Of the total 30 children, 14 had earlier seizures which were distinct from the later complex partial seizures (table 2). Three had prolonged febrile seizures lasting 30 minutes or more. Two had prolonged seizures not associated with fever in addition to short febrile seizures, and two had a short febrile seizure only. Seven further children had isolated non-febrile status epilepticus including one with a history of infantile spasms. In total, therefore, 12 children had suffered prolonged early seizures, and two a brief early febrile seizure.

Table 2 shows the location of the MRI determined abnormality as a function of a history of early seizures. Of the 16 with a hippocampal abnormality, 12 had suffered previous early seizures, including at least one episode of status epilepticus in 10 . Of the 16 children without a history of febrile and/or prolonged seizures there were only four children who had a hippocampal abnormality, and in none of these was it present without associated temporal abnormality.

\section{Discussion}

MRI can now define subtle abnormalities of the hippocampus and cortical grey matter. ${ }^{18} 20$ This study reviews the cerebral magnetic resonance images of 30 consecutive children aged $0 \cdot 9-15 \cdot 5$ years who presented to a tertiary referral centre with intractable complex partial seizures. The children included in this study were referred for the management of epilepsy which was difficult to control, not having responded to initial anticonvulsant treatment. Although at the time of study they were not necessarily under consideration for epilepsy surgery, two have since been operated upon and a surgical option is being evaluated in others as a result of the study findings. In this selected group of patients, many of whom are at the severe end of the clinical spectrum, all had identifiable MRI abnormalities. In 29 this abnormality was cerebral and in one it was cerebellar. This high rate of abnormality suggests that optimised MRI detects cortical and hippocampal grey matter lesions as well as foreign tissue lesions with a high degree of sensitivity. In a surgical temporal lobectomy series, which may reflect a more highly selected but similarly severe group of patients (in that there has been a poor response to medical treatment), a high rate of pathologically determined lesions was also found. ${ }^{12} 21$

Conventional EEG provided helpful information on lateralisation in $41 \%$. Specific localisation was made on the basis of the EEG in $31 \%$. This compares with the MRI identification of a focal imaging abnormality in $62 \%$. The increased sensitivity of MRI over EEG was found in children with complex partial seizures alone (33\% had localised EEG; $67 \%$ had a focal MRI abnormality) and those with complex partial seizures as part of a multiple seizure disorder (21\% had localised EEG; $57 \%$ a focal imaging abnormality). The low percentage of clear lateralisation and localisation on interictal and even ictal EEG in children is consistent with previous findings. ${ }^{22}$ Ictal recordings involve prolonged monitoring that can be difficult in children and presurgical evaluation may require invasive depth electrode recordings which are also more difficult in children than adults. This

Table 2 Location of MRI determined abnormality as a function of early childhood seizures

\begin{tabular}{llllll}
\hline & No of subjects & $\begin{array}{l}\text { Hippocampal } \\
\text { abnormality }\end{array}$ & $\begin{array}{l}\text { Hippocampal+ } \\
\text { temporal neocortical } \\
\text { abnormality }\end{array}$ & $\begin{array}{l}\text { Temporal neocortical } \\
\text { abnormality }\end{array}$ & $\begin{array}{l}\text { Extratemporal } \\
\text { abnormality }\end{array}$ \\
\hline Early childhood seizures & 14 & 5 & 7 & 2 & 0 \\
$\quad \begin{array}{l}\text { Short febrile convulsion } \\
\text { Febrile status }\end{array}$ & 2 & 0 & 2 & 0 & 0 \\
$\begin{array}{l}\text { Short febrile convulsion } \\
\text { non-febrile status }\end{array}$ & 3 & 0 & 2 & 1 & 0 \\
$\quad \begin{array}{l}\text { Non-febrile status } \\
\text { No early childhood seizures }\end{array}$ & 2 & 2 & 0 & 0 & 0 \\
\hline
\end{tabular}


difficulty in localisation techniques has been a limiting step in the assessment of children for epilepsy surgery. MRI now provides a means of identifying focal brain abnormalities not previously detectable by non-invasive means, and if the relationship between these structural abnormalities and ictal events can be established it offers a new approach to epilepsy surgery in children.

There is a strong correlation between the clinical localisation of temporal and extratemporal origin and the site of abnormality as revealed by MRI. All those with clinical temporal localisation and all but one of those without localising features had a temporal lobe abnormality on MRI. Only four children had extratemporal seizures. None of these children had abnormalities of the temporal lobe as determined by blinded MRI reporting.

Hippocampal abnormality was found in 16 of the total 30 children. This represents $62 \%$ if the four with clinical extratemporal epilepsy are excluded in order to provide a comparison to the frequency of hippocampal sclerosis found in surgical temporal lobectomy cases in adults. This percentage of hippocampal abnormality is similar to that found in adult surgical series. ${ }^{6}$ The presence of this high rate of MRI determined hippocampal abnormality indicates that this pathology is present early in the natural history of many who suffer temporal lobe epilepsy. The MRI finding of hippocampal sclerosis was present as early as 4 years of age in this series, which provides strong evidence that hippocampal sclerosis may be present from a very young age. The ability of MRI to detect lesions of the hippocampus at this early age means that such children have an identifiable structural abnormality which may allow early surgical treatment where appropriate, and also provides a means whereby the natural history of this lesion may be studied.

The identification of hippocampal sclerosis in these patients raises the question of how often it may be an incidental lesion, and how often it occurs secondary to seizures originating at another site. The relationship of these lesions, when found in young children, to the ictal focus has yet to be determined but in adults a strong association between the presence of a lesion, the site of the epileptogenic focus, and outcome after removal of the lesion has been shown. ${ }^{12425}$ Similar findings in children would have clear implications for the non-invasive preoperative assessment of children with focal epilepsy. The findings of this study provide the basis for future studies using MRI which can address these issues, and which can help to determine the relationship between early hippocampal damage and subsequent temporal lobe epilepsy.

A relationship between hippocampal sclerosis and early status epilepticus has been reported ${ }^{1526}$ and is also found in this series. The basis of this relationship is disputed, but it is clear from the current study that optimised MRI can contribute to investigation of this issue. Our findings, while supporting this association, cannot address the question of whether prolonged childhood seizures are caused by a pre-existing hippocampal abnormality, or whether hippo- campal sclerosis is the consequence of prolonged seizures. Progressive hippocampal abnormalities over a six month period were apparent in one child in the current series.

In $47 \%$ of our study group there was evidence of widespread cortical abnormalities. These abnormalities are often difficult to define precisely, and are of uncertain significance because of the difficulty in obtaining pathological correlation in these cases, but it is likely that they represent developmental abnormalities, neuronal migration abnormalities or diffuse cortical injury. In 11 cases (37\%) these abnormalities were present in addition to a more clearly defined focal lesion. It will be important to follow up these children to establish the prognostic significance of the widespread abnormalities, particularly their relevance to the problems of localisation by EEG and the sometimes poor results of surgical treatment..$^{24} 25$

This study relates the clinical features and antecedent history of seizures to the MRI findings. While pathological confirmation is only available in a few cases, the strong relationship between temporal lobe epilepsy and the finding of temporal or hippocampal abnormalities and the absence of these abnormalities in suspected extratemporal epilepsy, shows that intractable partial epilepsy is associated with specific structural lesions. The study provides evidence for the relationship between early status epilepticus and hippocampal sclerosis which has been suggested from earlier surgical series. ${ }^{27} \mathrm{MRI}$ provides an approach which can begin to separate the causes and the effects of seizures. It is hoped that this information will also allow appropriate early surgical intervention to alleviate the high psychiatric, social, and cognitive morbidity of severe uncontrolled epilepsy in childhood.

We thank Professor Jean Aicardi for helpful comments on earlier versions of the manuscript.

1 Harrison RM, Taylor DC. Childhood seizures: a 25-year follow up. Social and medical prognosis. Lancet 1976; i: 948 51.

2 Reynolds EH, Elwes RD, Shorvon SD. Why does epilepsy become intractable? Prevention of chronic epilepsy. Lancet 1983; ii: $952-4$.

3 Kotagal P, Rothner AD, Erenberg G, Cruse RP, Wyllie E. Complex partial seizures of childhood onset. A five year follow-up study. Arch Neurol 1987; 44: 1177-80.

4 Harbord MG, Manson JI. Temporal lobe epilepsy in childhood: reappraisal of etiology and outcome. Pediatr Neurol 1987; 3: 263-8.

5 Taylor DC. Mental state and temporal lobe epilepsy. A correlative account of 100 patients treated surgically. Epilepsia 1972; 13: 727-65.

6 Babb TL, Brown WJ. Pathological findings in epilepsy. In Engel J, ed. Surgical management of the epilepsies. New York: Raven Press, 1987: 511-40.

7 Bruton CJ. Neuropathology of the temporal lobe. Oxford University Press, 1988.

8 Janota I, Polkey CE. Cortical dysplasia in epilepsy - a study of material from surgical resections for intractable epilepsy. In Pedley TA, Meldrum BS, eds. Recent advances in epilepsy. No 5. Edinburgh: Churchill Livingstone, 1991: 37-49.

9 Duncan JS, Sagar HJ. Seizure characteristics, pathology, and outcome after temporal lobectomy. Neurology 1987; 37: Outcome.

10 Falconer MA, Serafetinides EA, Corsellis JAN. Etiology and pathogenesis of temporal lobe epilepsy. Arch Neurol 1964; 10: $233-40$

11 Cascino GD, Jack CR Jr, Parisi JE, et al. Magnetic resonance imaging in intractable frontal lobe epilepsy: pathologic 11: 51-9.

12 Jackson GD, Berkovic SF, Tress BM, Kalnins RM, Fabinyi GCA, Bladin PF. Hippocampal sclerosis can be reliably detected by magnetic resonance imaging. Neurology 1990 40: $1869-75$.

13 Kuzniecky R, Garcia JH, Faught E, Morawetz RB. Cortical dysplasia in temporal lobe epilepsy: magnetic resonance imaging correlations. Ann Neurol 1991; 29: 293-8. 
14 Cook MJ, Fish DR, Shorvon SD, Straughan K, Stevens JM. Hippocampal volumetric and morphometric studies in frontal and temporal lobe epilepsy. Brain 1992; 115: 100115.

15 Aicardi J, Chevrie JJ. Febrile convulsions. Sequelae and mental retardation. In: Brazier M, Coceani F, eds. Brain dysfunction in infantile febrile convulsions. International brain research organisation monograph series. New York: Raven research organisation m

16 Pampiglione G. Development of rhythmic EEG activities in infancy (waking state). Rev Electroencephalogr Neurophysiol infancy (waking state)

17 Shepherd JK, Hall-Craggs MA, Finn JP, Bingham RM. Sedation in children scanned with high field magnetic resonance; the experience at the Hospital for Sick Children, Great Ormond Street. Br f Radiol 1990; 63: 794-7.

18 Jackson GD, Berkovic SF, Duncan JS, Connelly A. Optimising the diagnosis of hippocampal sclerosis using magnetic resonance imaging. $A \mathcal{F N R}$ (in press).

19 Aicardi J. The agyria-pachygyria complex: a spectrum of cortical malformations. Brain Dev 1991; 13: 1-8.

20 Palmini AF, Andermann F, Olivier A, et al. Focal neuronal migration disorders and intractable partial epilepsy: a study of 30 patients. Ann Neurol 1991; 30: 741-9.

21 Duchowny $M$, Levin B, Jayakar P, et al. Temporal lobectomy in early childhood. Epilepsia 1992; 33: 298-303.

22 Holmes GL. Partial complex seizures in children: an analysis of 69 seizures in 24 patients using EEG FM radiotelemetry and videotape physiol 1984;57: 13-20.

23 Yamamoto N, Watanabe K, Negaro T, et al. Complex partial seizures in children: ictal manifestations and their relation to seizures in children: ictal manifestations and their relation to
clinical course. Neurology $1987 ; 37: 1379-82$.

24 Morris HH, Estes ML, Luders $\mathrm{H}$, et al. Electrophysiologic pathologic correlations in patients with complex partial seizures. Arch Neurol 1987; 44: 703-8.

25 Fish D, Andermann F, Olivier A. Complex partial seizures and small posterior temporal or extratemporal structura lesions: surgical management. Neurology 1991; 41: 1781-4.

26 Sagar HJ, Oxbury JM. Hippocampal neuronal loss in tempora lobe epilepsy: correlation with early childhood convulsions. Ann Neurol 1987; 22: 334-40.

27 Falconer MA. Genetic and related aetiological factors in temporal lobe epilepsy: a review. Epilepsia 1971; 12: 13-31. 